\title{
Study on Analysis of the MacKenzie Fall Sound
}

\author{
Zhixing Tian ${ }^{1}$, Ik-soo $\mathrm{Ahn}^{2}$ and Myung-Jin Bae ${ }^{3}$ \\ ${ }^{1}$ Soong-sil University, Department of Information and telecommunication Engineering, Seoul, 06978, Korea \\ Orcid Id : 0000-0003-3882-2459 \\ ${ }^{2}$ Soong-sil University, Department of Information and telecommunication Engineering, Seoul, 06978, Korea \\ Orcid Id : 0000-0003-1144-1963 \\ ${ }^{3}$ Soong-sil University, Department of Information and telecommunication Engineering, Seoul, 06978, Korea \\ Orcid Id : 0000-0002-7585-0400
}

\begin{abstract}
More people are beginning to pay attention to the psychological pleasure and relaxation brought by sound stimulation. In most cases, it seems that humans have a special preference for natural sounds. Natural sounds are mostly white noise and pink noise, such as wind, rain, sea waves and waterfall sounds, etc. They are all often considered to be beneficial to human health, but in fact, even natural sounds of the same category are also It will be very different because of space, time, and other factors. Each sound may be unique, so people's hearing experience is also different. This thesis quantitatively analyzes the sound spectrum and brain waves under natural sound stimulation. In this paper, the objective feeling of the MacKenzie Falls on the human auditory system is quantitatively analyzed through the sound spectrum and brain waves.
\end{abstract}

Keyword: Acoustics, EEG, Spectral Analysis, MacKenzie Falls Sound, Health, Brain Wave.

\section{INTRODUCTION}

The human auditory organs perceive sound mainly in three aspects: pitch, sound intensity and timbre. Pitch is the frequency of sound, and the human hearing frequency ranges from $20 \mathrm{~Hz}$ to $22 \mathrm{KHz}$. The audible frequency band bandwidth will gradually decrease with age. Humans have different objective perceptions of sounds at different frequencies in this frequency band. $20 \mathrm{~Hz}-60 \mathrm{~Hz}$ part: This section can give a strong, deep, and sensational feeling. $60 \mathrm{~Hz}-250 \mathrm{~Hz}$ part: This section is low frequency part of the sound, which can show a sense of rhythm. $50 \mathrm{~Hz}-4 \mathrm{KHz}$ part: This section is very close to the human voice, but it is a little dull. If it is too high, the sound will be similar to the sound on the phone. The human ear is more sensitive to this frequency band, but it is also more prone to hearing fatigue. $4 \mathrm{kHz}-5 \mathrm{KHz}$ part: This is the frequency band that affects the distance of the sound. High volume will make people feel that the sound source is very close to the listener; if the volume is too low, the distance of the sound will become farther, representing the energy part of the sound. $6 \mathrm{kHz}-20 \mathrm{kHz}$ part: This frequency band mainly represents the timbre, high volume makes the sound loud and bright, but not clear; low volume makes the sound clear, but slightly thin.[1] The human ear also perceives sound by frequency. There is a frequently curled basement membrane on the cochlea, and many auditory nerves are distributed under the basement membrane to receive sounds of different vibration frequencies. The basement membrane is divided into 25 frequency groups in the audible frequency band. Due to the masking effect, different frequency sounds in each same frequency group are superimposed together for frequency analysis. Figure 1 shows the structure of the cochlea flattened.[2] Sound intensity is the intensity of sound, that is, the magnitude of sound energy. The sound intensity range that human ears can feel is $10^{-16} \sim 10^{2} \mathrm{~W} / \mathrm{cm}^{2}$. If the sound intensity is too low, it will not be heard, and if it is too high, it will damage the hearing organs. Humans have different perceptions of sound intensity at different frequencies, that is, they have different psychological perceptions of the same sound intensity and different frequencies. The timbre is the unique feature of each sound. The brain distinguishes and recognizes the sound through the timbre, which is expressed as a spectrum envelope on the frequency spectrum. Therefore, the frequency spectrum analysis of the sound can obtain the frequency spectrum components and intensity of the sound and the timbre characteristics of the spectrum envelope. [3][4]

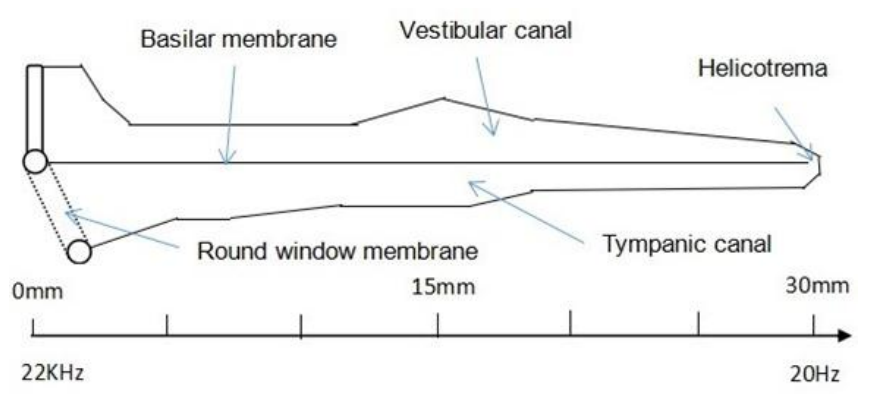

Figure 1. Flattened cochlear structure [2]

This paper discusses the sound of MacKenzie Falls. The MacKenzie Falls is the most iconic and spectacular waterfalls in Grampians Mountain, Australia. It is located in Grampians National Park. The waterfall is more than 60 meters highc At the bottom of the waterfall is a beautiful natural pool that connects the lower reaches of the MacKenzie River. Tourists can appreciate its beauty and listen to its sounds from different angles.[5] 
This thesis is divided into 5 parts. The first part has been briefly introduced. Chapter 2 is the spectrum analysis of the waterfall sound. Chapter 3 is the definition and analysis of brain waves. In chapter 4 , brain wave measurement experiment and brain wave analysis results. In chapter 5, the conclusion.

\section{SOUND SPECTRUM ANALYSIS}

The sound spectrum analysis tool is Adobe Audition CS6. The one-minute $44 \mathrm{khz}$ sampling frequency is subjected to FFT transformation with an FFT length of 1024 points, and the window function is the Hanning window. As shown in Figure 1 , the spectral energy of sound is widely distributed in the entire audible frequency band. The low-frequency energy is higher, and the spectral energy gradually decreases as the frequency increases. The spectral energy distribution characteristics are similar to pink noise ( $-3 \mathrm{~dB} /$ octave). Pink noise is considered a healthy sound favored by humans. In addition, there are three local peaks on the spectrogram, located at $500 \mathrm{~Hz}, 6 \mathrm{KHz}$, and $12 \mathrm{KHz}$. These three peaks determine the timbre characteristics of MacKenzie Falls sound. The first peak is located in the middle and low frequencies. It is the resonance sound of the waterfall's water falling from a high place to a low pool into a stream. The second peak is due to the sound of water flowing turbulently down the rock wall. The third peak is at a high frequency. This is because the sound of water falling from a height and hitting the surface of the water or rocks is very high. The low-frequency with high-energy sound is thick and deep, which can make people feel at ease and feel safe. And the highfrequency with high-energy sound is high-pitched and bright, which can improve people's alertness and concentration. [6] [7]

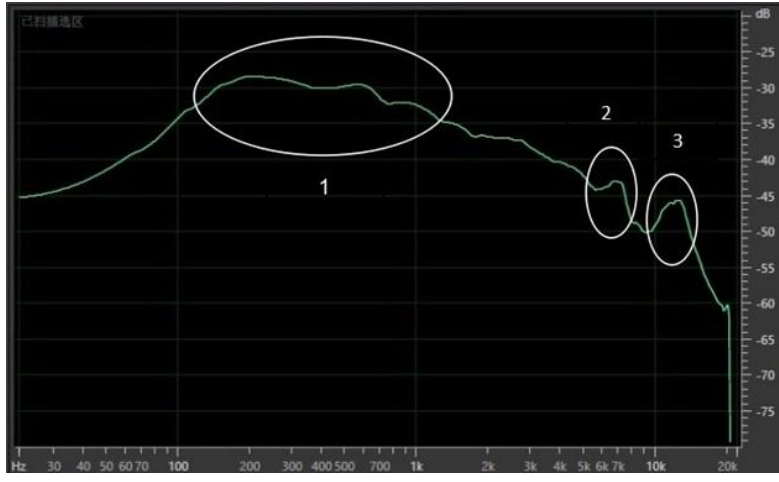

Figure 1. Spectrum analysis of MacKenzie Falls Sound

\section{DEFINITION OF BRAIN WAVE AND SPECTRUM ANALYSIS OF EEG}

\subsection{Definition of Brain Waves}

For human brain activity and emotion analysis, electroencephalogram (EEG) is usually used for analysis. It is an electrophysiological monitoring method for recording current brain electrical activity.[8] In other words, EEG records the changes in the time domain of potentials at different locations in the cerebral cortex. This time-domain signal is the sum of various chemical electrical activation signals, and it is difficult to directly analyze the time-domain signal. Therefore, in frequency analysis, through a large number of observation experiments, the brain waves are finally defined according to different frequencies and their functions. So far, brain waves are divided into 5 types, namely $\delta$ wave, $\theta$ wave, $\alpha$ wave, $\beta$ wave, and $\gamma$ wave.[9] But this experiment does not explain the $\gamma$ wave. Table 1 is the classification of brain waves and brain function.[10]

Table 1. Classification of brain waves [10]

\begin{tabular}{|c|c|c|}
\hline $\begin{array}{l}\text { Types } \\
\text { (Frequency/ } \\
\text { Hz) }\end{array}$ & $\begin{array}{c}\text { Amplitude } \\
\qquad(\mu \mathrm{V})\end{array}$ & Human Brain Activity \\
\hline $\begin{array}{c}\delta \text { wave } \\
(1-3)\end{array}$ & $20-200$ & $\begin{array}{l}\text { In most cases, it occurs in deep sleep. Neurons in the cortex are in a } \\
\text { state of simultaneous relaxation, which is an unconscious brain } \\
\text { wave. }\end{array}$ \\
\hline $\begin{array}{c}\theta \text { wave } \\
(4-7)\end{array}$ & $100-150$ & $\begin{array}{l}\text { It is a brain wave that appears between awake and sleep states. It is } \\
\text { the performance of the central nervous system's inhibition state, and } \\
\text { is related to the learning and memory processes. }\end{array}$ \\
\hline $\begin{array}{c}\alpha \text { wave } \\
(8-13)\end{array}$ & $20-100$ & $\begin{array}{l}\text { It is the main brain wave that appears in a normal quiet state, } \\
\text { indicating that in a quiet state, the cerebral cortical nerve cells are in } \\
\text { a ctate of relavation and nrenaration for artivity. The appearance of } \\
\text { a Wave can make people feel relaxed quickly. }\end{array}$ \\
\hline $\begin{array}{l}\beta \text { wave } \\
(14-30)\end{array}$ & $5-20$ & $\begin{array}{l}\text { It occurs when people perform behavioral actions and increase } \\
\text { attention, such as thinking about problems or performing } \\
\text { intellectual activities. However, the continuous emergence of a lot } \\
\text { of } \beta \text { waves will give a lot of pressure and tension. }\end{array}$ \\
\hline
\end{tabular}




\subsection{Spectrum Analysis of EEG Signal}

The EEG signal is a non-stationary signal, but it is stable in a short time. Therefore, the framing process should be performed before the spectrum analysis, and the feature extraction of the EEG signal is also based on the frame. The analysis methods for non-stationary signals usually include short-time Fourier transform, wavelet transform, Hilbert-Huang transform, linear prediction, and so on.[11] They can all reflect the instantaneous spectral characteristics of the signal and extract the characteristics of the signal. Then these extracted signals are classified and analyzed. Commonly used classification methods include K-nearest neighbor (KNN) classifier and support vector machine (SVM). These machine learning-based classification methods are too complicated because they require a lot of training data and a lot of calculations, and the tablebased classification method used in this paper comes from this paper.[12] Through frequency analysis of EEG, brain wave components and its quantity can be obtained, and finally, the proportion of each brain wave can be compared to judge the emotional state of the testee.

The spectrum analysis method used in this paper is power spectrum density analysis based on linear prediction.[12][13] Autocorrelation function (ACF) and the power spectral density function are a pair of Fourier transform. The following is the short-term autocorrelation function.

$$
R_{n}(k)=\sum_{m=-\infty}^{\infty} x(m) W(n-m) x(m+k) W(n-m+
$$$$
k)
$$

It can be defined like this.[14]

$$
\begin{gathered}
h_{k}=W(n) W(n+k) \\
R_{n}(k)=\sum_{m=-\infty}^{\infty} x(m) x(m-k) h_{k}(n-m) \\
=[x(m) x(m-k)] * h_{k}
\end{gathered}
$$

Therefore, the short-term autocorrelation function can be regarded as the output of the sequence $x(m) x(m-k)$ through the $h_{k}$ filter. Where the $\mathrm{W}(\mathrm{n})$ is FFT windows that is a hamming window with 256 points.

Because autocorrelation function and power spectral density function are Fourier transforms of each other.

$$
\begin{gathered}
F_{n}(K) \stackrel{D F T}{\Longleftrightarrow} R_{n}(k) \\
F_{n}(K)=\sum_{n=0}^{N-1} R_{n}(k) e^{-j \frac{2 \pi}{N} K n}
\end{gathered}
$$

Consider passing an AR filter to get the spectral envelope of the power spectrum, where $\mathrm{G}$ is the gain and $\mathrm{p}$ is the order.

$$
H(z)=\frac{\mathrm{G}}{1-\sum_{i=1}^{p} a_{i} z^{-i}}
$$

The relationship between input $y(n)$ and output $x(n)$ is expressed by a difference equation. [13]

$$
y(n)=\sum_{i=1}^{p} a_{i} x(n-i)+G x(n)
$$

Let $G x(n)$ be the prediction error, and define the difference equation of the linear predictor as:

$$
\overline{y(n)}=\sum_{i=1}^{p} a_{i} x(n-i)
$$

The transfer function of the $\mathrm{P}$-order predictor. [13]

$$
P(z)=\sum_{i=1}^{p} a_{i} z^{-i}
$$

Use the autocorrelation method to solve the prediction coefficients, the following is the minimizing Yule-Walker equation and error function. [13]

$$
\begin{gathered}
\sum_{i=0}^{p} a_{i} r(|j-r|)=r(j) \quad 1 \leq j \leq p \\
E=r(0)-\sum_{i=1}^{p} a_{i} r(i) \quad(12)
\end{gathered}
$$

Express (11) as a Toeplitz matrix

$$
\begin{gathered}
{\left[\begin{array}{rlcc}
R(0) & R(-1) & \ldots & R(-p+1) \\
R(1) & R(0) & & R(-p+2) \\
& \vdots & \ddots & \vdots \\
R(p-2) & R(p-3) & & R(-1) \\
R(p-1) & R(p-2) & \ldots & R(0)
\end{array}\right]\left[\begin{array}{c}
a(1) \\
a(2) \\
\vdots \\
a(p-1) \\
a(p)
\end{array}\right]} \\
=\left[\begin{array}{c}
R(1) \\
R(2) \\
\vdots \\
R(p-1) \\
R(p)
\end{array}\right]
\end{gathered}
$$

Finally, the prediction coefficient, a(.) can be solved by the Levinson-Durbin recursive algorithm. [13]

\section{EXPERIMENTS AND RESULTS}

\subsection{Experimental Process}

First, record the sound source of MacKenzie fall for one minute and save it in wave format. Then four college students with normal hearing between 20 and 25 years old were measured brain waves, two men, and two women. The test instrument used in the experiment is a two-channel, $45 \mathrm{~Hz}$ bandwidth brainwave measuring instrument produced by NeuroHarmory, s. Single channel sampling frequency is $256 \mathrm{~Hz}$. The experimental site is a quiet laboratory.

In order to compare the brain wave changes caused by sound stimulation, the experiment is divided into two parts. The first part measures the brain waves of the testee in a quiet environment without any sound. The second part measures the brain waves of the testee when receiving sound stimulation. The brainwave measurement time for each part is 2 minutes and 30 seconds. All of testees are supposed to keep quiet and closed 
their eyes. In order to eliminate the psychological impact of physiological activities, the test was performed one hour after meals. Finally, EEG signal data is obtained. [14]

\subsection{Experimental Results and Simulation}

The EEG signal with a frame length of 4 seconds at one minute in the measurement experiment was intercepted for spectrum analysis. Figures 3, 4, 5, and 6 show the PSD and spectrograms of the EEG of four subjects before and after listening. Each
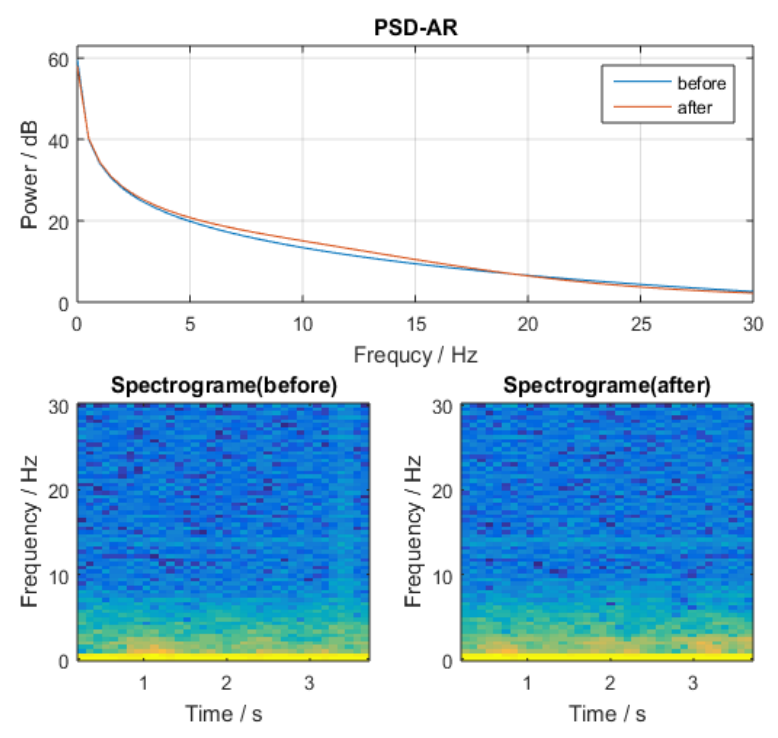

Figure 3. Test result of testee A
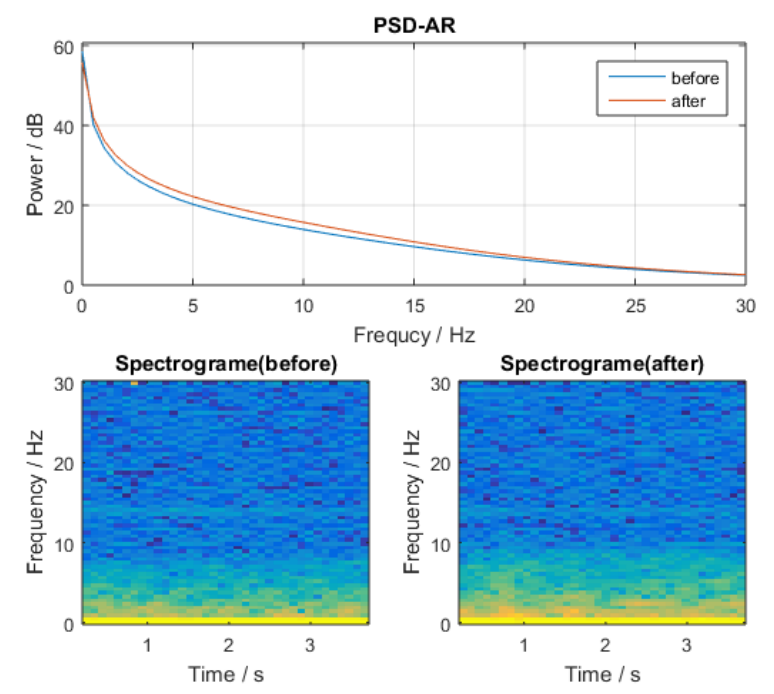

Figure 4. Test result of testee B picture shows the test result of a subject. It can be seen from the PSD diagram that when listening to the waterfalls sound, it is increasing that the $\alpha$ brain waves of four testees. For $\beta$ brain waves, Testee D's $\beta$ brain waves are significantly reduced, and there is no difference for others. There is no obvious difference between the $\delta$ brain waves and $\theta$ brain waves of all of the testees before and after sound stimulation. It can be seen from the spectrogram that the yellow energy area of the spectrogram becomes wider when the sound is stimulated, which means that the energy of the $\alpha$ brain wave area increases, and there are more $\alpha$ brain waves. [12] [14] [15]
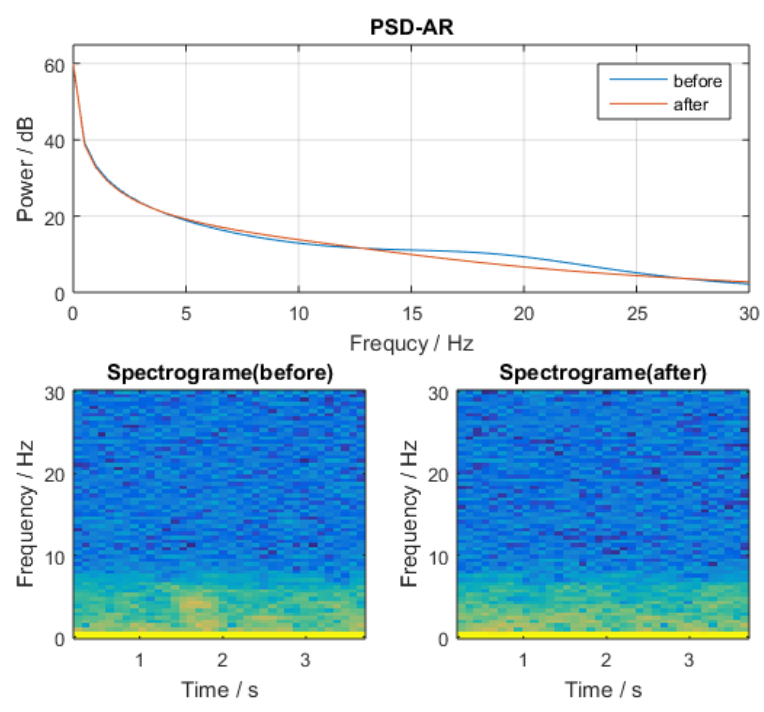

Figure 5. Test result of testee C
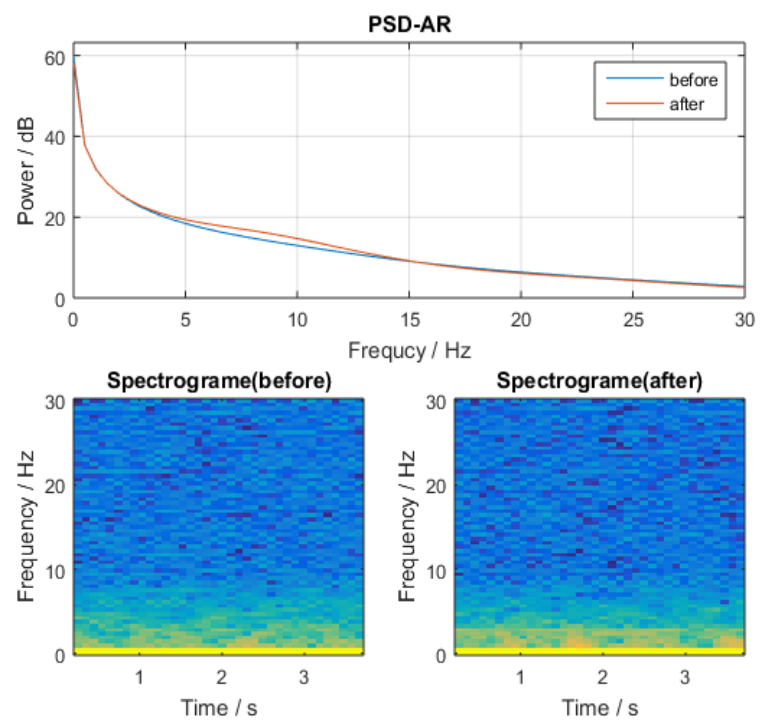

Figure 6. Test result of testee D 
In summary, the short-term EEG spectrum analysis before and after sound stimulation is analyzed. Next, the brainwave components of the EEG signal in all measurement periods will be averaged and analyzed. In order to determine the proportion of various brain waves in the total duration of the test. Figure 7 shows the percentages of various brain waves before and after sound stimulation. It can be seen that the brain waves of the four testees have the same trend after sound stimulation. That is, the $\delta$ brain waves decrease, the $\theta$ brain waves have no obvious changes, and the $\alpha$ brain waves increase. The $\beta$ brain waves of $A$ and $B$ remain unchanged, while the $\beta$ brain waves of $C$ and $D$ increase. In short, their $\alpha$ brain waves have increased significantly, and their $\delta$ brain waves have decreased. A lot of $\alpha$ brain waves can bring relaxation, relieve stress and tension, and promote the body to enter a quiet state of rest. This is a brain wave that is good for health and can relieve fatigue. However, the MacKenzie Falls sound can promote this brain wave. [16] [17]

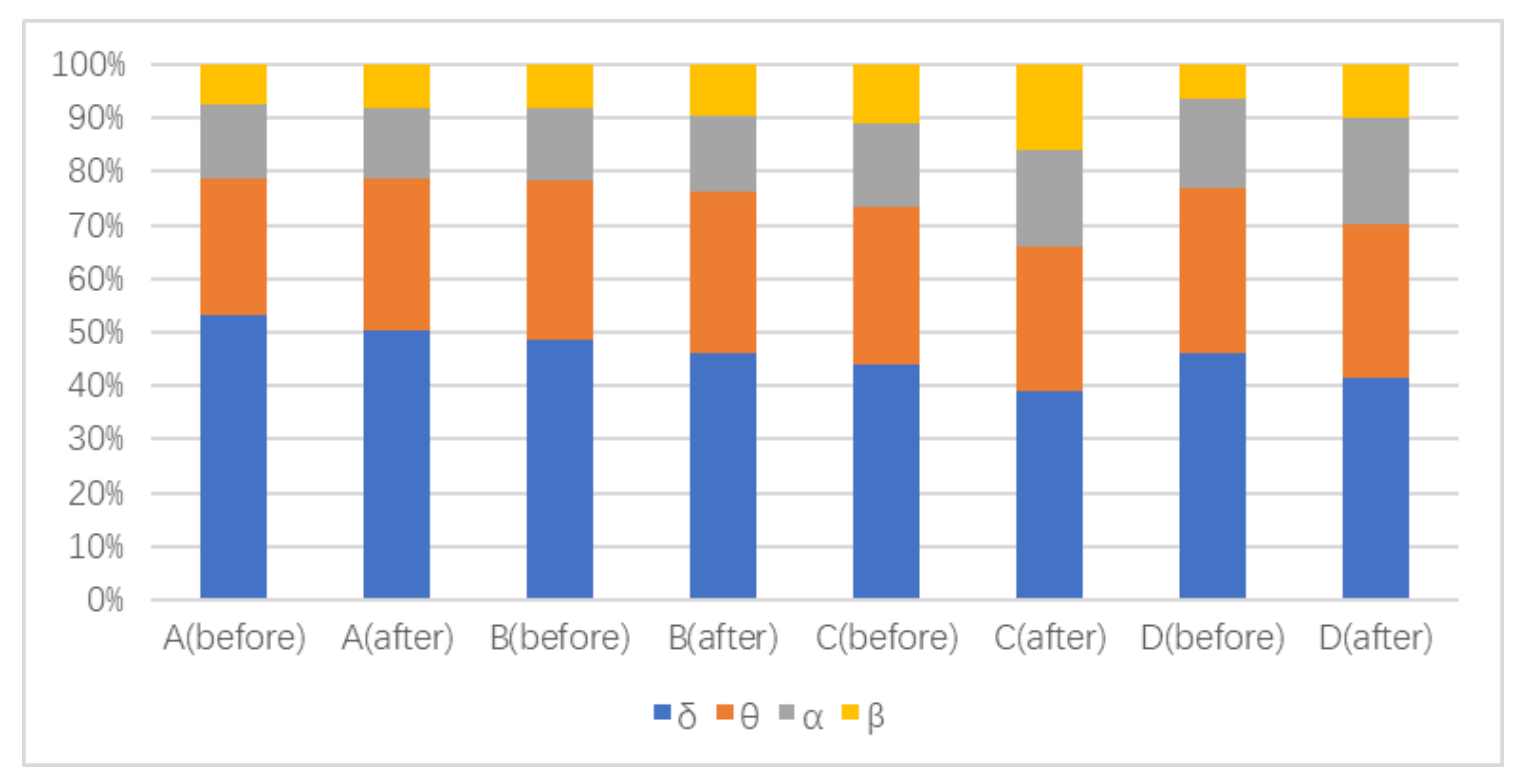

Figure 7. The percentage of brain waves of the four subjects before and after hearing the MacKenzie Falls sound

\section{CONCLUSION}

This paper analyzes the sound composition of the MacKenzie Falls from the perspective of frequency based on the way humans perceive sound, and explains its acoustic characteristics. In order to further study the response of the human brain to the MacKenzie Falls sound stimulation, the EEG signal was analyzed by frequency spectrum, and the brain wave components and content of the EEG signal were obtained. And we compare the results before and after stimulation.

From the analysis result of the audio frequency domain, the overall spectral energy distribution characteristics are similar to pink noise. But it has three distinct local peaks, located at low, mid, and high frequencies. This is the result of the resonance of the sound of the water flowing with the surrounding environment caused by the special structure of the waterfall. Brainwave analysis is performed by using PSD based on linear prediction. The results show that listening to that sound can induce more $\alpha$ waves while suppressing $\delta$ waves. Therefore, it can be judged that the Mackenzie Falls sound can relieve fatigue and stress, relax the body, and actively promote people's health. In the following research, we will study more methods to analyze EEG signals under sound stimulation, such as wavelet transform and Hilbert-Huang transform.

\section{REFERENCE}

[1] Baidu experience, The characteristics of sound in different frequency bands of popular science, [https://jingyan.baidu.com/article/948f5924469935990ef 5f913.html]

[2] SungTae Lee, Principle and Application of Sound, Chungmoongak Press, 2010.

[3] Ik-Soo Ahn, Bong-young kim, and Myung-jin Bae. "A study on the human sensation of the reed wind sound in ASMR," International Journal of Engineering Research and Technology, Volume 12, Issue 9, Pages 1494-1499, 2019.

[4] Myung-Jin Bae, Digital Speech Communications. Dongyung Press Co., 2010.

[5] Parks VICTORIA, MACKENZIE FALLS, 2020, [https://www.parks.vic.gov.au/places-tosee/parks/grampians-national-park/attractions/centralgrampians/things-to-do/mackenzie-falls].

[6] Zhixing Tian, Bong-Young Kim, and Myung-Jin Bae. "Study on the acoustic characteristics of Sunwapta Falls," International Journal of Engineering Research and Technology, Volume 13, Issue 5, pp.962-966, 2020.

[7] Zhixing Tian, Bong-Young Kim, and Myung-Jin Bae, "Study on acoustic analysis of Cleveland Dam waterfalls 
sound," International Journal of Engineering Research and Technology, Volume 13, Issue 6, pp.1159-1164, 2020.

[8] Wikipedia, Electroencephalography, 2020, [https://en.wikipedia.org/wiki/Electroencephalography].

[9] Y. Sun, N. Ye, X. Wang, and X. Xu," The Research of EEG Analysis Methods Based on Sounds of Different Frequency," 2007 IEEE/ICME International Conference on Complex Medical Engineering, Beijing, pp.17461751, 2007.

[10] Personal library, The four brains of human, [http://www.360doc.com/content/15/0714/17/15991787_ 484900161.shtml].

[11] Zhengqiang Ni, Lei Wang, Jun Meng, Fuming Qiu, and Jian Huang, "EEG Signal Processing in Anesthesia Feature Extraction of Time and Frequency parameters," Procedia Environmental Sciences 8, pp 215-220, 2011.

[12] Kwang-Bock You, Zhixing Tian, and Myung-Jin Bae, “A Study on the Characteristics of EEG Signals by Auditory Stimulation of the Nature Sounds," International Journal of Engineering Research and Technology, Vol.13, No.8, To be published, 2020.
[13] Song Zhiyong, MATLAB Speech Signal Analysis and Synthesis (Second Edition), BEIHANG UNIVERSITY PRESS. Jan. 2018.

[14] Zhixing Tian, Ik-soo Ahn, and Myung-Jin Bae, "Study on the Health Application of the Snow Stepping Sound," International Journal of Engineering Research and Technology, Vol.13, No.7, To be published, August 2020.

[15] Ik-Soo Ahn, Bong-Young Kim, and Kwang-Bock You, Myung-Jin Bae, "A Study on the Characteristics of an EEG Based on a Singing Bowl's Sound Frequency," Studies in Computational Intelligence, Volume 789, Pages 233-243, 2019.

[16] Kyoung-su Yeo, Myungsook Kim, and Myung-Jin Bae, "Acoustic characteristics of the forest sounds inducing sleep," Information (Japan), Volume 18, Issue 10, Pages 4407-4412, October 2015.

[17] Semion Kizhner, Thomas P. Flatley, Norden E. Huanh, Karin Blank, and Evette Conwell, "On the Hilbert-Huang Transform Data Processing Systems Development," IEEE, 2014 IEEE Aerospace Conference Proceedings, pp.1961-1979, 2014. 